\title{
Silencing of polo-like kinase 2 increases cell proliferation and decreases apoptosis in SGC-7901 gastric cancer cells
}

\author{
LI YING LIU ${ }^{1 *}$, WEI WANG ${ }^{2 *}$, LING YU ZHAO ${ }^{1}$, BO GUO $^{1}$, JUAN YANG $^{1,3}$, \\ XIAO GE ZHAO ${ }^{1}$, TU SHENG SONG ${ }^{3}$, CHEN HUANG ${ }^{1,3}$ and JI RU XU ${ }^{4}$ \\ ${ }^{1}$ Center Laboratory of Biomedical Research, Key Laboratory of Environment and Genes Related to Diseases of \\ Chinese Ministry of Education, Xi'an Jiaotong University Health Science Center, Xi'an, Shaanxi 710061; \\ ${ }^{2}$ Department of Orthopaedic Surgery, The Second Affiliated Hospital of Xi'an Jiaotong University, Xi'an, \\ Shaanxi 710004; Departments of ${ }^{3}$ Genetics and Molecular Biology, and ${ }^{4}$ Immunology and Pathogenic Biology, \\ Molecular Bacteriology Laboratory, Key Laboratory of Environment and Genes Related to Diseases of \\ Chinese Ministry of Education, Xi'an Jiaotong University Health Science Center, \\ Xi'an, Shaanxi 710061, P.R. China
}

Received February 27, 2014; Accepted November 19, 2014

DOI: $10.3892 / \mathrm{mmr} .2014 .3077$

\begin{abstract}
Polo-like kinase 2 (PLK2) is a serine/threonine protein kinase, which has vital roles during mitosis and the centrosome cycle. In acute myeloblastic leukemia and hepatocarcinogenesis, PLK2 acts as a tumor suppressor; however, the function of PLK2 in gastric cancer remains to be elucidated. In the present study, PLK2 was overexpressed in gastric cancer tissues and three types of gastric cancer cells, SGC-7901, MKN-45 and BGC-823. Transfection of SGC-7901 gastric cancer cells with small interfering (si)RNA against PLK2 exerted no effect on the ratio of cells at different stages of the cell cycle compared with that of the untransfected and control siRNA-transfected cells. In addition, silencing of PLK2 significantly enhanced the growth of SGC-7901 cells through inhibiting apoptosis. Furthermore, apoptosis-associated genes Bax and caspase 3 were found to be downregulated at the
\end{abstract}

Correspondence to: Professor Ji Ru Xu, Department of Immunology and Pathogenic Biology, Molecular Bacteriology Laboratory, Key Laboratory of Environment and Genes Related to Diseases of Chinese Ministry of Education, Xi'an Jiaotong University Health Science Center, 76 West Yanta Road, Xi'an, Shaanxi 710061, P.R. China

E-mail: xujiru@mail.xjtu.edu.cn

Professor Chen Huang, Center Laboratory of Biomedical Research, Key Laboratory of Environment and Genes Related to Diseases of Chinese Ministry of Education, Xi'an Jiaotong University Health Science Center, 76 West Yanta Road, Xi'an, Shaanxi 710061,P.R. China E-mail: hchen@mail.xjtu.edu.cn

${ }^{*}$ Contributed equally

Key words: polo-like kinase 2, small interfering RNA, proliferation, apoptosis, SGC-7901 cells protein level. In conclusion, these results suggested that PLK2 may act as a tumor suppressor in gastric cancer, therefore indicating its therapeutic potential.

\section{Introduction}

Gastric cancer, also known as stomach cancer, has a high incidence rate in and is one of the most prominent causes of cancer-associated mortality in Asia (1). In addition, gastric cancer is the fourth most prevalent type of cancer worldwide and the second most frequent cause of cancer-associated mortality worldwide $(2,3)$. The prognosis of gastric cancer patients is poor and it remains challenging to cure. Targeted gene therapy is a novel therapeutic approach for gastric cancer, for which the identification of tumor suppressors associated with this malignancy is essential.

Polo-like kinase 2 (PLK2) is a member of the serine/threonine protein kinase family, which includes five members: PLK1, PLK2 (also termed SNK), PLK3 (also termed Fnk or Prk), PLK4 (also termed Sak) and PLK5 (4-6). These kinases have important roles during mitosis and the centrosome cycle (7). PLK2 is located in the centrosome and was found to be involved in embryonic development and cell cycle progression at the $\mathrm{G}_{1} / \mathrm{S}$ transition, as well as in skeletal development. Cell cycle analysis of cultured PLK2 ${ }^{-/}$embryonic fibroblasts indicated that these cells proliferated more slowly than cells expressing PLK2 and exhibited delayed entry into $S$ phase from $G_{1}(8)$. In central neurons, PLK2 was reported to be overexpressed in response to synaptic stimulation (9). Decreased expression of PLK2 was observed in B-cell malignancies; in addition, apoptosis was found to be induced in Burkitt's lymphoma cells through the ectopic expression of PLK2 (10). This therefore indicated that PLK2 may act as a tumor suppressor gene in hematologic malignancies $(10,11)$. Pellegrino et al (12) showed that PLK2 was a tumor suppressor in hepatocarcinogenesis. In addition, Kothari et al (13) reported that PLK2 is an outlier kinase, which was highly expressed in 
KRAS-dependent pancreatic cancer cells. The MIA-PaCa-2 pancreatic cancer cell line was used to assess the effects of small interfering (si)RNA silencing of PLK2 on cell proliferation; the results showed significant growth inhibition (13), suggesting that PLK2 may have an oncogenic role in pancreatic cancer and therefore may have comparable effects in different types of cancer. However, the expression and function of PLK2 in gastric cancer remains to be elucidated. In the present study, siRNA mediated knock-down of PLK2 in SGC-7901 gastric cancer cells was used to examine the expression and activity of PLK2 in gastric cancer.

\section{Materials and methods}

Collection of clinical samples. A total of 24 gastric cancer tissue samples and adjacent normal tissues were collected from patients undergoing surgery for gastric cancer at the First and Second Affiliated Hospitals of the Medical College of Xi'an Jiaotong University (Shaanxi, China). Samples were placed in liquid nitrogen as soon as they were obtained and then stored at $-80^{\circ} \mathrm{C}$ until RNA extraction. The study was approved by the ethics committee of Xi'an Jiatong University Health Science Centre (Xi'an, China). Written informed consent was obtained from all patients or their families.

Cell culture and transfection. GES-1 human fetal gastric epithelial cells and SGC-7901, BGC-823, AGS and MKN-45 gastric cancer cells were obtained from The Central Laboratory of Biomedical Research of the Medical College of Xi'an Jiaotong University. Cells were cultured in Dulbecco's modified Eagle's medium (DMEM; Gibco-BRL, Carlsbad, CA, USA) with $10 \%$ fetal bovine serum (FBS; Gibco-BRL), $100 \mathrm{U} / \mathrm{ml}$ penicillin and $100 \mu \mathrm{g} / \mathrm{ml}$ streptomycin (Invitrogen Life Technologies, Carlsbad, CA, USA), and grown in a $37^{\circ} \mathrm{C}$ incubator with $5 \% \mathrm{CO}_{2}$. siRNAs targeting PLK2 (5'-GAGCAGCUGAGCACAUCAUDTDT-3') and the silencer negative controls (siN05815122147) were purchased from Guangzhou RiboBio Co, Ltd (Guangzhou, China). Lipofectamine $^{\circledR} 2000$ (Invitrogen Life Technologies) was used to transfect PLK2 siRNA and control siRNA into SGC-7901 cells at a final concentration of $50 \mathrm{nM}$ in all experiments, according to the manufacturer's instructions. Cells were then harvested $24 \mathrm{~h}$ post-transfection. The expression of PLK2 mRNA was detected in the SGC-7901, BGC-823, AGS and MKN-45 gastric cancer cells lines and the control GES-1 cell line, whereas in subsequent experiments only the SGC-7901 cell line was analyzed.

$R N A$ extraction and reverse transcription-quantitative polymerase chain reaction ( $R T-q P C R)$ analyses. RNA was extracted using TRIzol ${ }^{\circledR}$ (Invitrogen Life Technologies) according to the manufacturer's instructions. Total RNA was used to generate cDNA using a PrimeScript RT reagent kit (Takara Bio. Inc., Shiga, Japan). The program used for reverse transcription was as follows: $37^{\circ} \mathrm{C}$ for $15 \mathrm{~min}, 85^{\circ} \mathrm{C}$ for $5 \mathrm{sec}$. Then the cDNA was used as a template for qPCR. PLK2 expression in gastric cancer samples and gastric cancer cells was assessed using qPCR with SYBR premix Ex Taq $^{\mathrm{TM}}$ II (Takara) on a Roche 480 Light cycler (Roche Diagnostics $\mathrm{GmbH}$, Basel, Switzerland) according to the manufacturer's instructions. Primer sequences (Primer Premier 5.0; Premier Biosoft, Palo Alto, CA, USA) were as follows: Forward: 5'-AATAACTCAGCAACCCAGCAAAC-3' and reverse: 5'-GTGACCCACTGAAATGATGTGC-3' for PLK2; and forward: 5'-GAAGGTGAAGGTCGGAGTC-3' and reverse: 5'-GAAGATGGTGATGGGATTTC-3' for GAPDH, as used by Radonića et al (14). The PCR program used for amplification was as follows: $94^{\circ} \mathrm{C}$ for $3 \mathrm{~min}$, followed by 40 cycles of $94^{\circ} \mathrm{C}$ for $20 \mathrm{sec}, 60^{\circ} \mathrm{C}$ for $40 \mathrm{sec}$ and $72^{\circ} \mathrm{C}$ for $40 \mathrm{sec}$. qPCR results were analyzed using the $2^{-\Delta \Delta \mathrm{Ct}}$ method.

MTT assay. A cell proliferation assay, using thiazolyl blue (Sigma-Aldrich, St. Louis, MO, USA) was performed in 96-well culture plates. A total of $5 \times 10^{4}$ cells $/ \mathrm{ml}$ were seeded into each well and grown at $37^{\circ} \mathrm{C}$ in $5 \% \mathrm{CO}_{2}$ for 3 days. The growth medium was replaced with serum-free medium prior to transfection. MTT solution ( $5 \mathrm{mg} / \mathrm{ml}$; Sigma-Aldrich) was added to each well at 24, 48 and $72 \mathrm{~h}$ and cells were then incubated at $37^{\circ} \mathrm{C}$ for $3 \mathrm{~h}$. Following removal of the liquid supernatant, $0.2 \mathrm{ml}$ dimethyl sulfoxide (Sigma-Aldrich) was added to each well. Optical density was determined at $490 \mathrm{~nm}$ using a FLUOstar/POLARstar OPTIMA (BMG LABTECH $\mathrm{GmbH}$, Ortenberg, Germany). Data represent the results of experiments performed at least in triplicate.

Cell cycle analysis. A total of $1 \times 10^{5}$ cells/well were plated onto six-well plates and transfected with PLK2 siRNA and control siRNA. Cells were harvested at $24 \mathrm{~h}$ post-transfection, washed with cold phosphate-buffered saline (PBS) and fixed in ice-cold $70 \%$ alcohol at $4^{\circ} \mathrm{C}$ overnight. Fixed cells were washed twice in PBS, then resuspended in $0.5 \mathrm{ml}$ PBS containing propidium iodide (PI; $50 \mu \mathrm{g} / \mathrm{ml}$ ) and RNase A (200 $\mu \mathrm{g} / \mathrm{ml}$; Amresco LLC, Solon, OH, USA) for $30 \mathrm{~min}$ at room temperature in the dark. Cell cycle analysis was then performed using a FACSArray (Becton Dickinson, Franklin Lakes, NJ, USA).

Cell apoptosis analysis. A total of $1 \times 10^{5}$ cells/well were plated onto a six-well plate and transfected with PLK2 siRNA and control siRNA. Cells were harvested at $24 \mathrm{~h}$ post-transfection, washed twice with PBS and then resuspended in $0.5 \mathrm{ml}$ buffer solution (Roche Diagnostics, Shanghai, China) containing PI and Annexin V (Roche Diagnostics) at a final concentration of $1 \mu \mathrm{g} / \mathrm{ml}$, and incubated for $30 \mathrm{~min}$ at room temperature in the dark. A FACSArray (Becton Dickinson) was then used to calculate cell apoptosis rates.

Western blot analysis. Cells (1×105/well) were plated onto a six-well plate and transfected with PLK2 siRNA and control siRNA. Cells were then harvested at $24 \mathrm{~h}$ post-transfection, washed with PBS and lysed using radioimmunoprecipitation assay lysis buffer (Beyotime Institute of Biotechnology, Shanghai, China). Proteins were separated on 10\% SDS polyacrylamide gels and transferred onto polyvinylidene fluoride (PVDF) membranes (Roche Diagnostics GmbH) through electroblotting. Membranes were blocked with 5\% non-fat milk and probed with primary antibodies against human PLK2 (Snk/H90, sc-25421; Santa Cruz Biotechnology, Inc., Dallas, TX, USA), GAPDH (6C5, sc-32233; Santa Cruz Biotechnology,Inc.), Bax (sc-493; ZSGB-BIO, Beijing, China), 
Table I. Antibody details.

\begin{tabular}{lccccc}
\hline Gene & Catalog number & Animal raised in & Animal raised against & Dilution & Mono/polyclonal \\
\hline PLK2 & sc-25421 & rabbit & human & $1: 500$ & polyclonal \\
GAPDH & sc-32233 & mouse & human & $1: 1000$ & monoclonal \\
Bax & sc-493 & rabbit & human & polyclonal \\
CDK2 & ab2363 & mouse & human & $1: 100$ & monoclonal \\
caspase 3 & sc-65497 & mouse & human & $1: 500$ & monoclonal \\
$\beta$-actin & KC-5A08 & mouse & & $1: 5000$ & monoclonal \\
\hline
\end{tabular}
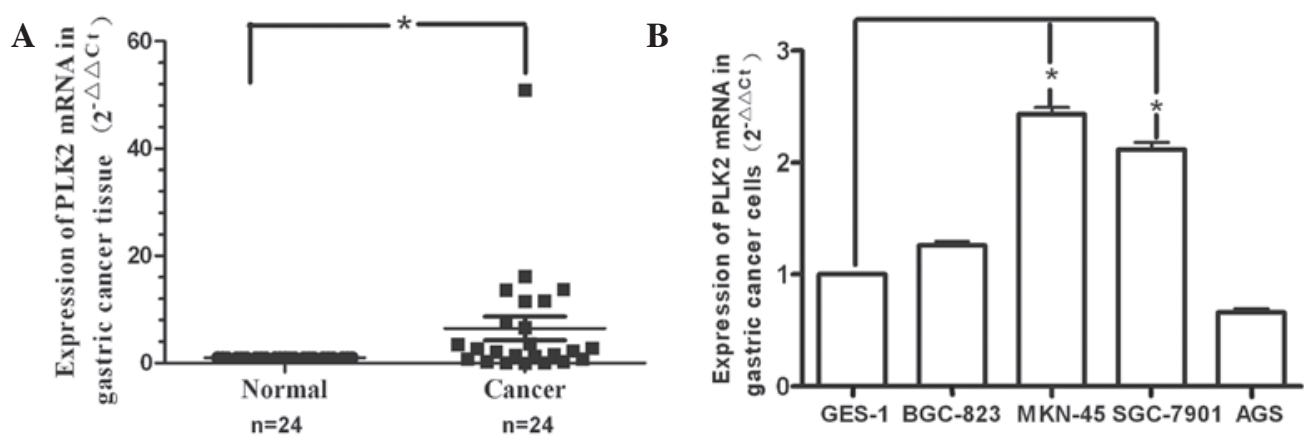

Figure 1.PLK2 mRNA expression levels in gastric cancer tissue and gastric cancer cells. (A) PLK2 mRNA levels in gastric cancer tissue and adjacent normal tissue were quantified using RT-qPCR and normalized to that of the housekeeping gene GAPDH. (B) PLK2 mRNA levels in BCG-823, MKN-45, SGC-7901 and AGS gastric cancer cell lines as well as GES-1 cells were quantified using RT-qPCR and normalized to that of the housekeeping gene GAPDH. "P<0.05 vs. normal tissue or GES-1 cells. PLK2, polo-like kinase 2; GES-1, human fetal gastric epithelial cells; RT-qPCR, reverse transcription-quantitative polymerase chain reaction.

caspase-3 (sc-65497; ZSGB-BIO), CDK2 (ab2363; Abcam, Cambridge, MA, USA), and $\beta$-actin (KC-5A08; KangChen Bio-tech, Shanghai, China) (Table I). The membranes were further probed with horseradish peroxidase-conjugated rabbit anti-mouse and goat anti-rabbit secondary antibodies (ZDR-5109 and ZDR-5118; 1:50; ZSGB-BIO). Working solutions of the Enhanced Chemiluminescence Substrate (Pierce Biotechnology, Inc., Rockford, IL, USA) were prepared and added to PVDF membranes for $1 \mathrm{~min}$. The membranes were then removed from the substrates and exposed to ChemiDoc-It 510 (UVP, LLC, Upland, CA, USA).

Statistical analysis. Statistical analysis was performed using SPSS 13.0 software (SPSS, Inc., Chicago, IL, USA). P-values were calculated using a one-way analysis of variance. $\mathrm{P}<0.05$ was considered to indicate a statistically significant difference between values.

\section{Results}

Expression of PLK2 mRNA in gastric cancer samples and gastric cancer cells. A total of 24 matched gastric cancer samples and adjacent normal tissues were collected from patients with gastric cancer. As shown in Fig. 1A, RT-qPCR revealed that PLK2 expression was significantly increased in 17 of the 24 tumor samples compared with that of the corresponding normal tissue. The expression levels of PLK2 varied among the 24 gastric cancer patients; however, a general trend towards increased expression in gastric cancer tissues was observed. In BGC-823, MKN-45 and SGC-7901 gastric cancer cell lines

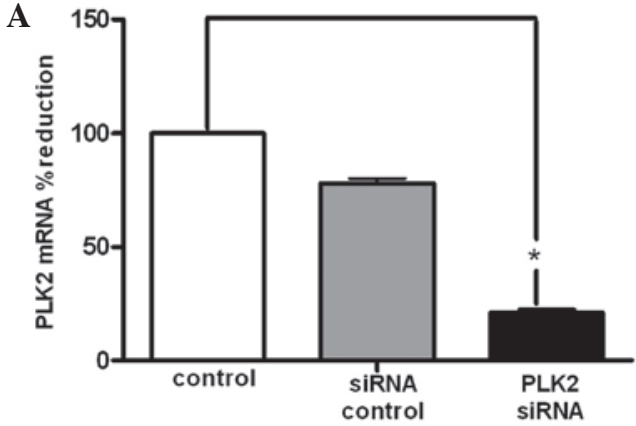

B

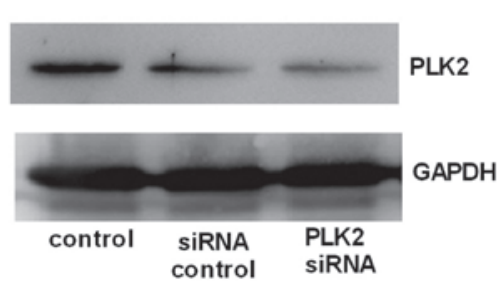

Figure 2. PLK2 expression in SGC-7901 gastric cancer cells following transfection with PLK2 siRNA. PLK2 siRNA, siRNA control at $50 \mathrm{nM}$ or Lipofectamine $2000^{\circledR}$ only (control) were transfected into SGC-7901 cells for $24 \mathrm{~h}$. (A) PLK2 mRNA levels were quantified using reverse transcription-quantitative polymerase chain reaction. (B) PLK2 protein expression were determined by western blot analysis. Values were normalized to that of the housekeeping gene GAPDH. " $\mathrm{P}<0.05$, compared with control. PLK2, polo-like kinase 2; siRNA, small interfering RNA.

(Fig. 1B), PLK2 mRNA expression was increased to varying degrees compared with that of the GES-1 cells; however, only MKN-45 and SGC-7901 cells showed a significant increase. By contrast, PLK2 expression was downregulated in AGS cells. 

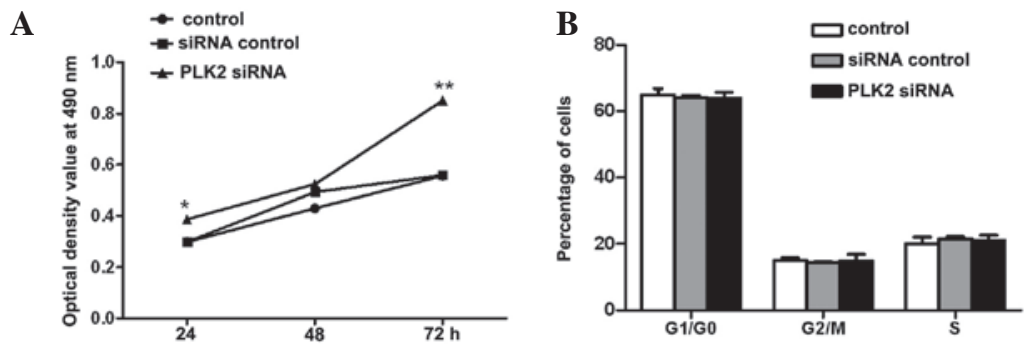

C

D
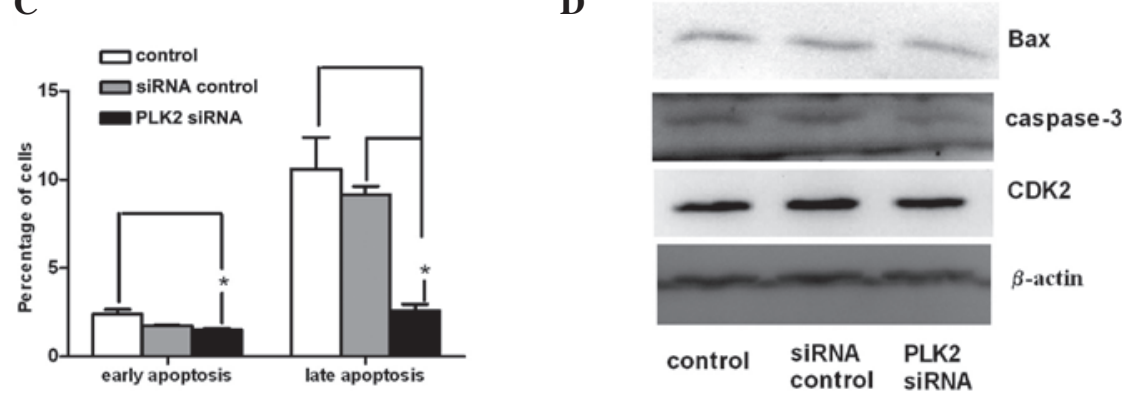

Figure 3. Effects of PLK2 siRNA on proliferation, cell cycle and apoptosis of SGC-7901 cells. SGC-7901 cells were treated with PLK2 siRNA, siRNA control at $50 \mathrm{nM}$ or Lipofectamine $2000^{\circledast}$ only (control). (A) SGC-7901 cell viability was measured using the MTT assay at 24, 48 and $72 \mathrm{~h}$ post-transfection. (B) Cell cycle progression was analyzed using cell cycle kinetics $24 \mathrm{~h}$ post-transfection. Cells were stained with propidium iodide and analyzed using FACS. (C) FACS analysis was also used to determine the apoptotic rate of SGC-7901 cells at $24 \mathrm{~h}$ post-transfection. (D) Western blot analysis was used to determine the levels of apoptosis-associated proteins, Bax and caspase-3, and the cell cycle-related protein CDK2 in SGC-7901 cells 24 h post-transfection. "P<0.05 vs. control. PLK2, polo-like kinase 2; siRNA, small interfering RNA; FACS, fluorescence activated cell sorting; CDK, cyclin-dependent kinase.

PLK2 interference effect. Following transfection of SGC-7901 cells with PLK2 siRNA for $24 \mathrm{~h}$, the cells were collected and RNA and protein were extracted. As shown in Fig. 2A, compared with the untransfected and control siRNA control groups, PLK2 mRNA expression was significantly decreased in the PLK2 siRNA group. In addition, the results of the western blot analysis were consistent with those of the RT-qPCR, therefore demonstrating that PLK2 protein levels were reduced in the PLK2 siRNA group (Fig. 2B).

PLK2 siRNA promotes the growth of SGC-7901 cells. An MTT assay was used to examine the effects of PLK2 siRNA on SGC-7901 cell viability. The growth of SGC-7901 cells was detected for three days following transfection with PLK2 siRNA. As shown in Fig. 3A, compared with the untransfected and siRNA control groups, PLK2 siRNA significantly promoted the growth of SGC-7901 cells at 24 and $72 \mathrm{~h}$. This result was consistent with the findings previously reported by Li et al (15), which demonstrated that the viability of granulosa cells increased significantly following PLK2 siRNA treatment and suggested that PLK2 expression blocks granulosa cell proliferation (15). Pellegrino et al (12) also showed that PLK2 inactivation led to increased cell viability.

PLK2 siRNA has no effect on SGC-7901 cell cycle progression. PLK2 is activated close to the G1-to-S phase transition of the cell cycle (16); therefore, the effect of PLK2 silencing on cell cycle progression was investigated in the present study. Cell cycle analysis was performed $24 \mathrm{~h}$ following treatment with PLK2 siRNA (Fig. 3B); however, no significant changes in the ratio of cells at each stage of the cell cycle were observed among groups at $24 \mathrm{~h}$. These results differ from those reported by Li et al (15), which showed that PLK2 siRNA reduced the percentage of cells in the G0/G1 phase as well as increased the percentage of cells in the G2 phase and S phase. Ma et al (8) investigated how cell cycle progression was affected by PLK2 deletion, the results of which showed that FACS analysis of DNA content revealed a higher number of PLK2 ${ }^{+/}$cells than $\mathrm{PLK}^{-/-}$cells in S phase. These data suggested that PLK2 may influence G1 progression; however, unlike PLK1, it is not required for cell division.

PLK2 siRNA decreases apoptosis of SGC-7901 cells. The results of the Annexin-V/PI assay showed that the apoptosis of SGC-7901 cells decreased significantly following PLK2 siRNA treatment for $24 \mathrm{~h}$ (Fig. 3C). The percentage of early apoptotic cells in the PLK2 siRNA group was $1.52 \%$, which was slightly lower than that of the other two groups at $24 \mathrm{~h}$; however, this was only significantly lower than the untreated control. By contrast, the percentage of late apoptotic cells in the PLK2 siRNA group was significantly decreased compared with that of the two control groups at $24 \mathrm{~h}$. The percentages of apoptotic cells in the control group, siRNA control group and PLK2 siRNA group were 10.6, 9.14 and 2.61\%, respectively. These findings suggested that PLK2 overexpression may induce apoptosis in SGC-7901 cells. In 2006, Syed et al (10) demonstrated that overexpression of PLK2 in B cell lymphomas led to apoptosis (10). From the results shown in Fig. 3C in the present study, it was inferred that PLK2 inhibited SGC-7901 cell growth through the induction of apoptosis.

Western blot analysis of apoptosis-associated proteins. Due to the obvious effect of PLK2 on apoptosis, the expression of apoptosis-associated proteins, Bax and caspase-3, was examined in SGC-7901 cells. The results revealed that PLK2 siRNA downregulated the expression of Bax and caspase 3 (Fig. 3D). 
However, expression levels of the cell cycle-associated protein CDK2 were not altered in response to PLK2 siRNA (Fig. 3D). These results were consistent with those of the cell cycle analysis.

\section{Discussion}

PLK2 is a member of the polo-like kinase family, the members of which have previously been reported to regulate the cell cycle as well as DNA damage-induced checkpoints in mammals $(10,11)$. PLK2 was classified as an early growth-response gene due to its increased expression following growth factor stimulation (17). PLK2, as a proliferation-associated gene, has been investigated in association with tumor treatment. In different tumors, PLK2 was found to have a dual role as an oncogene or tumor suppressor gene. In the present study, the expression of PLK2 was examined in gastric cancer and gastric cancer cells. Among the 24 matched gastric cancer samples, 17 demonstrated PLK2 overexpression. In addition, PLK2 expression was found to be upregulated in three gastric cancer cell lines, including BGC-823, MKN-45 and SGC-7901. These result indicated that PLK2 was overexpressed in the majority of the gastric cancer samples examined as well as in gastric cancer cells. The expression levels of PLK2 may be associated with altered tumor pathological classification and staging; however, future studies are required in order to further examine this.

In the present study, in order to explore the role of PLK2 in gastric cancer, the behavior of SGC-7901 cells was investigated in response to PLK2 siRNA transfection. Cell cycle analysis showed no differences among the number of cells in G0/G1, S and G2/M phases between the PLK2 siRNA group and the two control groups. These results were comparable with those reported by Burns et al (18), in which no significant differences were observed in the cell cycle at each phase between U20S, H460 and HeLa cells transfected with PLK2 siRNA and controls. This therefore suggested that PLK2 was not required for normal progression through the cell cycle and mitosis. Strebhardt (19) reported that the cell cycle profiles and fractions of cells with sub-G1 DNA content were not altered following an siRNA-mediated decrease in PLK2 expression in different cell lines; whereas, in the present study, siRNA-mediated silencing of PLK2 improved the growth of SGC-7901 cells through decreasing apoptosis. These results therefore indicated that SGC-7901 cell proliferation was not mediated by an effect of PLK2 on the cell cycle but rather through decreasing apoptosis. The results of western blot analysis of apoptosis-associated genes were consistent with those of the cell cycle analysis. The cell cycle-associated CDK2 protein expression was not altered and Bax and caspase-3 were slightly downregulated.

Notably, in the present study, PLK2 functioned as a tumor suppressor in gastric cancer and its expression was upregulated. However, these findings were inconsistent with a previous study that demonstrated high gene expression levels of PLK1 or PLK2 have been observed in pancreatic cancer (12). Conversely, in a previous study, PLK2 overexpression in B cell lymphomas was shown to lead to apoptosis (10). Similarly, PLK2 acted as a tumor suppressor in hepatocellular carcinoma, in which its expression was significantly decreased (11). Gene expres- sion in cancer is regulated by oncogenes and tumor suppressor genes. In recent years, with the identification of microRNAs (miRNAs) and their functions, Li et al (20) and Miko et al (21) reported that PLK2 was a target gene of miR126. miRNAs are a type of small non-coding RNA, 22-nucleotides, which downregulate the translation of target mRNAs $(22,23)$. miR126 was first identified in a tissue specific mouse screen (24) and was encoded by intron 7 of the EGF-like domain 7 gene in mammals and birds $(25,26)$. miR-126 is downregulated and acts as a tumor suppressor in stomach cancer (27). A previous study by our group also found that miR-126 acted as a tumor suppressor in gastric carcinoma, and PLK2 was a target gene of miR-126 (28). This therefore indicated that the upregulation of PLK2 in gastric cancer may be associated with the downregulation of miR-126; thus, miR-126 may also have a dominant role in gastric cancer. However, further studies are required in order to elucidate the role of miR-126 in gastric cancer.

In conclusion, the results of the present study demonstrated that PLK2 functioned as a tumor inhibitor in gastric cancer and may therefore have potential for development as a novel gene therapy in gastric cancer.

\section{Acknowledgements}

The present study was supported by a grant from the Fundamental Research Funds for the Central Universities of Xi'an Jiaotong University (no. 08143014).

\section{References}

1. Jemal A, Murray T, Ward E, et al: Cancer statistics, 2005. CA Cancer J Clin 55: 10-30, 2005.

2. Parkin DM, Bray FL and Devesa SS: Cancer burden in the year 2000. The global picture. Eur J Cancer 37: 4-66, 2001.

3. Parkin DM: International variation. Oncogene 23: 6329-6340, 2004.

4. Glover DM, Hagan IM and Tavares AA: Polo-like kinases: a team that plays throughout mitosis. Gene Dev 12: 3777-3787, 1998.

5. Barr FA, Sillje HH and Nigg EA: Polo-like kinases and the orchestration of cell division. Nat Rev Mol Cell Biol 5: 429-440, 2004.

6. Andrysik Z, Bernstein WZ, Deng L, et al: The novel mouse polo-like kinase 5 responds to DNA damage and localizes in the nucleolus. Nucleic Acids Res 38: 2931-2943, 2010.

7. Archambault V and Glover DM: Polo-like kinases: conservation and divergence in their functions and regulation. Nat Rev Mol Cell Biol 10: 265-275, 2009

8. Ma S,Charron J,Erikson RL et al: Role of plk2 (Snk) in mouse development and cell proliferation. Mol Cell Biol 23: 6936-6943, 2003.

9. Kauselmann G, Weiler M, Wulff P, et al: The polo-like protein kinases Fnk and Snk associate with a $\mathrm{Ca}(2+)$ - and integrin-binding protein and are regulated dynamically with synaptic plasticity. EMBO J 18: 5528-5539, 1999.

10. Syed N, Smith P, Sullivan A, et al: Transcriptional silencing of polo-like kinase 2 (SNK/PLK2) is a frequent event in B-cell malignancies. Blood 107: 250-256, 2006.

11. Smith P, Syed N and Crook T: Epigenetic inactivation implies a tumor suppressor function in hematologic malignancies for Polo-like kinase 2 but not Polo-like kinase 3. Cell Cycle 5: 1262-1264, 2006.

12. Pellegrino R, Calvisi DF, Ladu S, et al: Oncogenic and tumor suppressive roles of polo-like kinases in human hepatocellular carcinoma. Hepatology 51: 857-868, 2010.

13. Kothari V, Wei I, Shankar S, et al: Outlier kinase expression by RNA sequencing as targets for precision therapy. Cancer Discov 3: 280-293, 2013.

14. Radonića A, Thulkea S, Mackayb IM, et al: Guideline to reference gene selection for quantitative real-time PCR. Biochem Biophys Res Common 313: 856-862, 2004. 
15. Li F, Jo M, Curry TE Jr and Liu J: Hormonal induction of polo-like kinases (Plks) and impact of Plk2 on cell cycle progression in the rat ovary. PLoS One 7: e41-e44, 2012.

16. Warnke S, Kemmler S, Hames RS, et al: Polo-like kinase-2 is required for centriole duplication in mammalian cells. Curr Biol 14: 1200-1207, 2004

17. Simmons DL, Neel BG, Stevens R, et al: Identification of an early-growth-response gene encoding a novel putative protein kinase. Mol Cell Biol 12: 4164-4169, 1992.

18. Burns TF, Fei P, Scata KA, et al: Silencing of the novel p53 target gene Snk/Plk2 leads to mitotic catastrophe in paclitaxel (taxol)-exposed cells. Mol Cell Biol 23: 5556-5571, 2003.

19. Strebhardt K: Multifaceted polo-like kinases: drug targets and antitargets for cancer therapy. Nat Rev Drug Discov 9: 643-660, 2010.

20. Li Z, Lu J, Sun M, et al: Distinct microRNA expression profiles in acute myeloid leukemia with common translocations. Proc Natl Acad Sci USA 105:15535-15540, 2008.

21. Miko E, Margitai Z, Czimmerer Z, et al: miR-126 inhibits proliferation of small cell lung cancer cells by targeting SLC7A5. FEBS Lett 585: 1191-1196, 2011.
22. Lee RC, Feinbaum RL and Ambros V: The C. elegans heterochronic gene lin-4 encodes small RNAs with antisense complementarity to lin-14. Cell 75: 843-854, 1993.

23. Esquela-Kerscher A and Slack FJ: Oncomirs-microRNAs with a role in cancer. Nat Rev Cancer 6: 259-269, 2006.

24. Lagos-Quintana M, Rauhut R, Yalcin A, et al: Identification of tissue-specific microRNAs from mouse. Curr Biol 12: 735-739, 2002.

25. Wang S, Aurora AB, Johnson BA, et al: The endothelial-specific microRNA miR-126 governs vascular integrity and angiogenesis. Dev Cell 15: 261-271, 2008.

26. Fish JE, Santoro MM, Morton SU, et al: miR-126 regulates angiogenic signaling and vascular integrity. Dev Cell 15: 272-284, 2008.

27. Feng R, Chen X, Yu Y, et al: miR-126 functions as a tumour suppressor in human gastric cancer. Cancer Lett 298: 50-63, 2010.

28. Liu LY, Wang W, Zhao LY, et al: miR-126 inhibits growth of SGC-7901 cells by synergistically targeting the oncogenes PI3KR2 and Crk, and the tumor suppressor PLK2. Int J Oncol 45: 1257-1265, 2014. 